Research Article

\title{
On the Norms of RFMLR-Circulant Matrices with the Exponential and Trigonometric Functions
}

\author{
Baijuan Shi iD \\ School of Science, Xi'an University of Posts and Telecommunications, Xi'an, Shaanxi, China \\ Correspondence should be addressed to Baijuan Shi; 593800425@qq.com
}

Received 29 April 2021; Revised 5 June 2021; Accepted 8 June 2021; Published 17 June 2021

Academic Editor: Ghulam Shabbir

Copyright ( 2021 Baijuan Shi. This is an open access article distributed under the Creative Commons Attribution License, which permits unrestricted use, distribution, and reproduction in any medium, provided the original work is properly cited.

In this paper, based on combinatorial methods and the structure of RFMLR-circulant matrices, we study the spectral norms of RFMLR-circulant matrices involving exponential forms and trigonometric functions. Firstly, we give some properties of exponential forms and trigonometric functions. Furthermore, we study Frobenius norms, the lower and upper bounds for the spectral norms of RFMLR-circulant matrices involving exponential forms and trigonometric functions by some ingenious algebra methods, and then we obtain new refined results.

\section{Introduction}

Matrix analysis theory is a powerful tool to study modern communication systems; especially, matrix norm is very important for neural network-based adaptive tracking control for switched nonlinear systems [1]. Recently, studying the norms of special matrices has been a hot topic in matrix theory. Especially, some scholars studied the norms of $r$-circulant matrices, geometric circulant matrices, and $r$-Hankel and $r$-Toeplitz matrices with some famous numbers and polynomials. For example, on the spectral norms of circulant matrices, $r$-circulant matrices, geometric circulant matrices, and $r$-Hankel and $r$-Toeplitz matrices with Fibonacci number, Lucas number, generalized Fibonacci and Lucas numbers, and generalized $k$-Horadam numbers have been studied [2-8]. We have obtained several results $[9,10]$ of the norms of matrices mentioned above with exponential forms $e(k / n)$ and trigonometric functions $\cos (k \pi / n)$ and $\sin (k \pi / n)$. There is a RFMLRcirculant matrix applied first by Jiang [11]. As far as we know, it seems that no one has studied the upper and lower estimate problems for the spectral norms of RFMLR-circulant matrices involving exponential forms $e(k / n)$ and trigonometric functions $\cos (k \pi / n)$ and $\sin (k \pi / n)$ yet. Although some scholars at home and abroad have given algorithms about norms of circulant matrices, the computational complexity of these algorithms is amazing with the increase of matrix order. To overcome this defect, we have constructed matrix factorization and then we study the norms of RFMLR-circulant matrices involving exponential forms and trigonometric functions. Compared with the existing methods, the algorithm model of this study is easy to implement and then we use some ingenious methods to obtain new refined results. All results can be well applied in adaptive feedback control, and these have potential applications in neural network nonlinear systembased norms.

For $e(x), \quad e(x)=e^{2 \pi i x}$ and then $|e(x)|=1$; by $e^{i \theta}=\cos \theta+i \sin \theta$, note that $e(0)=e(1)=e(-1)=$ $e(n)=1$, and by the trigonometric sums, we have

$$
\sum_{k=1}^{n} e\left(\frac{k m}{n}\right)= \begin{cases}n, & n \mid m, \\ 0, & \text { otherwise. }\end{cases}
$$

Particularly, $\quad \sum_{k=0}^{n-1} e(k / n)=0, \quad \cos \theta=\left(e^{i \theta}+e^{-i \theta}\right) / 2$ and $\sin \theta=\left(e^{i \theta}-e^{-i \theta}\right) / 2$. By the relationship between exponential forms $e(k / n)$ and trigonometric functions $\cos (k \pi / n)$ and $\sin (k \pi / n)$, we can obtain some power sums of these functions.

A $n \times n$ row first-minus-last right- (RFMLR-) circulant matrix with the first row $\left(a_{0}, a_{1}, \ldots, a_{n-1}\right)$, denoted by $A=\operatorname{RFMLRcircfr}\left(a_{0}, a_{1}, \ldots, a_{n-1}\right)$, is defined by [11] 
$A=\left(\begin{array}{cccccc}a_{0} & a_{1} & a_{2} & \cdots & a_{n-2} & a_{n-1} \\ a_{n-1} & a_{0}-a_{n-1} & a_{1} & \cdots & a_{n-3} & a_{n-2} \\ a_{n-2} & a_{n-1}-a_{n-2} & a_{0}-a_{n-1} & \cdots & a_{n-4} & a_{n-3} \\ \vdots & \vdots & \vdots & & \vdots & \vdots \\ a_{1} & a_{2}-a_{1} & a_{3}-a_{2} & \cdots & a_{n-1}-a_{n-2} & a_{0}-a_{n-1}\end{array}\right)_{n \times n}$

Obviously, the RFMLR-circulant matrix is determined by its first row, and we define $\Theta_{(1,-1)}$ as the basic RFMLRcirculant matrix with the first row $(0,1,0, \ldots, 0)$, namely,

$$
\Theta_{(1,-1)}=\left(\begin{array}{ccccc}
0 & 1 & 0 & \cdots & 0 \\
0 & 0 & 1 & \cdots & 0 \\
\vdots & \vdots & \vdots & & \vdots \\
1 & -1 & 0 & \cdots & 0
\end{array}\right)_{n \times n}
$$

We can obtain $\Theta_{(1,-1)}^{n}=I_{n}-\Theta_{(1,-1)}$. According to the structure of the power of the basic RFMLR-circulant matrix $\Theta_{(1,-1)}$, it is clear that

$$
A=\operatorname{RFMLRcircfr}\left(a_{0}, a_{1}, \ldots, a_{n-1}\right)=\sum_{i=0}^{n-1} a_{i} \Theta_{(1,-1)}^{i} .
$$

Inspired by reference [11], based on preliminary work, in this paper, we shall use identities of exponential forms $e(k / n)$ and trigonometric functions $\cos (k \pi / n)$ and $\sin (k \pi / n)$ and power sums of $e(k / n), \cos (k \pi / n)$, and $\sin (k \pi / n)$ to study the norms of RFMLR-circulant matrices:

$$
\begin{aligned}
& A=\operatorname{RFMLRcircfr}\left(e\left(\frac{0}{n}\right), e\left(\frac{1}{n}\right), e\left(\frac{3}{n}\right), \ldots, e\left(\frac{n-1}{n}\right)\right), \\
& B=\operatorname{RFMLRcircfr}\left(\cos \left(\frac{0 \cdot \pi}{n}\right), \cos \left(\frac{1 \cdot \pi}{n}\right), \cos \left(\frac{2 \cdot \pi}{n}\right), \ldots, \cos \left(\frac{(n-1) \cdot \pi}{n}\right)\right), \\
& C=\operatorname{RFMLRcircfr}\left(\sin \left(\frac{0 \cdot \pi}{n}\right), \sin \left(\frac{1 \cdot \pi}{n}\right), \sin \left(\frac{2 \cdot \pi}{n}\right), \ldots, \sin \left(\frac{(n-1) \cdot \pi}{n}\right)\right) .
\end{aligned}
$$

We give Frobenius norms, the lower and upper bounds for the spectral norms of these matrices. Some interesting and concise results are stated by the following theorems.

Theorem 1. Let

$$
A=\operatorname{RFMLRcircfr}\left(e\left(\frac{0}{n}\right), e\left(\frac{1}{n}\right), e\left(\frac{3}{n}\right), \ldots, e\left(\frac{n-1}{n}\right)\right),
$$

be a $n \times n$ RFMLR-circulant matrix; then, we have

$$
\sqrt{\frac{n+1}{2}+2(n-1) \sin ^{2}\left(\frac{\pi}{n}\right)} \leq\|A\|_{2} \leq 2 n-1 .
$$

Theorem 2. Let

$$
B=\operatorname{RFMLRcircfr}\left(\cos \left(\frac{0 \cdot \pi}{n}\right), \cos \left(\frac{1 \cdot \pi}{n}\right), \cos \left(\frac{2 \cdot \pi}{n}\right), \ldots, \cos \left(\frac{(n-1) \cdot \pi}{n}\right)\right)
$$

be a $n \times n$ RFMLR-circulant matrix; then, we have the following:

If $n$ is even,

$$
\sqrt{\frac{\alpha}{n}} \leq\|B\|_{2} \leq 2 \csc \left(\frac{\pi}{2 n}\right)-1,
$$

where

$$
\sqrt{\frac{\alpha}{n}} \leq\|B\|_{2} \leq 2 \cot \left(\frac{\pi}{2 n}\right)-1 .
$$

If $n$ is odd,

$$
\alpha=\frac{5 n^{2}-8 n+4}{4}+\frac{1}{4} \sec \left(\frac{\pi}{n}\right)-\left(n^{2}-6 n+6\right) \cos \left(\frac{\pi}{n}\right) .
$$

Theorem 3. Let 


$$
C=\operatorname{RFMLRcircfr}\left(\sin \left(\frac{0 \cdot \pi}{n}\right), \sin \left(\frac{1 \cdot \pi}{n}\right), \sin \left(\frac{2 \cdot \pi}{n}\right), \ldots, \sin \left(\frac{(n-1) \cdot \pi}{n}\right)\right)
$$

be a $n \times n$ RFMLR-circulant matrix, we have

$$
\sqrt{\frac{\beta}{n}}\|C\|_{2} \leq 2 \cot \left(\frac{\pi}{2 n}\right)
$$

where

$$
\beta=\frac{2 n^{2}+3 n-2}{4}-\frac{1}{8} \sec \left(\frac{\pi}{n}\right)
$$

\section{Preliminaries}

Definition 1 (see [9]). Let any matrix $A=\left(a_{i j}\right) \in M_{m \times n}(C)$; the spectral norm and the Euclidean norm of matrix $A$ are defined by

$$
\begin{aligned}
\|A\|_{2} & =\sqrt{\max _{1 \leq i \leq n} \lambda_{i}\left(A^{H} A\right),} \\
\|A\|_{F} & =\left(\sum_{i=1}^{m} \sum_{j=1}^{n}\left|a_{i j}\right|^{2}\right)^{(1 / 2)},
\end{aligned}
$$

respectively, where $\lambda_{i}\left(A^{H} A\right)$ is the eigenvalues of matrices $A^{H} A$ and $A^{H}$ is the conjugate transpose of $A$.

The following important inequalities hold between the Frobenius norm and spectral norm:

$$
\frac{1}{\sqrt{n}}\|A\|_{F} \leq\|A\|_{2} \leq\|A\|_{F}
$$

Lemma 1. For exponential forms $e(k / n)$,

$$
\begin{array}{r}
\sum_{k=0}^{n-1}\left|e\left(\frac{k}{n}\right)\right|=n, \\
\sum_{k=0}^{n-1} e\left(\frac{k}{n}\right)=\sum_{k=0}^{n-1} e\left(\frac{-k}{n}\right)=\sum_{k=0}^{n-1} e^{2}\left(\frac{k}{n}\right)=0 .
\end{array}
$$

Proof. Using the definition of $e(x), e(x)=e^{2 \pi i x}$ and $e^{i \theta}=\cos \theta+i \sin \theta$, we have $|e(x)|=1$, namely, $\sum_{k=0}^{n-1}|e(k / n)|=n$.

$e(k / n)$ is a geometric sequence, the common ratio is $e(1 / n)$, and $e(x) e(y)=e(x+y)$, so we have

$$
\sum_{k=0}^{n-1} e\left(\frac{k}{n}\right)=\sum_{k=0}^{n-1} e\left(\frac{-k}{n}\right)=\sum_{k=0}^{n-1} e^{2}\left(\frac{k}{n}\right)=0 .
$$

Lemma 2. For any positive integer $n \geq 2$, we have

$$
\begin{aligned}
& \sum_{k=0}^{n-1} \cos \left(\frac{k \pi}{n}\right)=1 \\
& \sum_{k=0}^{n-1} \sin \left(\frac{k \pi}{n}\right)=\cot \left(\frac{\pi}{2 n}\right) \\
& \sum_{k=0}^{n-1} \cos ^{2}\left(\frac{k \pi}{n}\right)=\sum_{k=0}^{n-1} \sin ^{2}\left(\frac{k \pi}{n}\right)=\frac{n}{2} .
\end{aligned}
$$

Proof. By the relationship between exponential forms and trigonometric functions, $\cos \theta=\left(e^{i \theta}+e^{-i \theta}\right) / 2$ and $\sin \theta=$ $\left(e^{i \theta}-e^{-i \theta}\right) / 2$, and using Lemma 1 , we can obtain sums mentioned above.

Lemma 3. If $n$ is even,

$$
\sum_{j=1}^{n-1}\left|\cos \left(\frac{j \pi}{n}\right)\right|=\cot \left(\frac{\pi}{2 n}\right)-1
$$

If $n$ is odd,

$$
\sum_{j=1}^{n-1}\left|\cos \left(\frac{j \pi}{n}\right)\right|=\csc \left(\frac{\pi}{2 n}\right)-1 .
$$

Proof. If $n$ is even,

$$
\begin{aligned}
\sum_{j=0}^{n-1}\left|\cos \left(\frac{j \pi}{n}\right)\right| & =2 \sum_{j=1}^{(n / 2)} \cos \left(\frac{j \pi}{n}\right)+1=2 \sum_{j=0}^{(n / 2)-1} \cos \left(\frac{j \pi}{n}\right)-1, \\
\sum_{j=0}^{(n / 2)-1} \cos \left(\frac{j \pi}{n}\right) & =\sum_{j=0}^{(n / 2)-1} \frac{e(j / 2 n)+e(-j / 2 n)}{2}=\frac{1}{2}\left(\frac{1-e(1 / 4)}{1-e(1 / 2 n)}+\frac{1-e(-1 / 4)}{1-e(-1 / 2 n)}\right) \\
& =\frac{1}{2} \frac{2-e(1 / 4)-e(-1 / 4)-e(1 / 2 n)-e(-1 / 2 n)+e((1 / 2 n)-(1 / 4))+e((1 / 4)-(1 / 2 n))}{2-e(1 / 2 n)-e(-1 / 2 n)} \\
& =\frac{1}{2} \frac{1-\cos (\pi / n)+\sin (\pi / n)}{1-\cos (\pi / n)} \\
& =\frac{1}{2}\left(1+\cot \left(\frac{\pi}{2 n}\right)\right) .
\end{aligned}
$$


If $n$ is odd,

$$
\begin{aligned}
\sum_{j=0}^{n-1}\left|\cos \left(\frac{j \pi}{n}\right)\right| & =\sum_{j=1}^{n-1}\left|\cos \left(\frac{j \pi}{n}\right)\right|+1=2 \sum_{j=1}^{(n-1) / 2} \cos \left(\frac{j \pi}{n}\right)+1 \\
& =2 \sum_{j=0}^{(n-1) / 2} \cos \left(\frac{j \pi}{n}\right)-1 \\
& =\sum_{j=0}^{(n-1) / 2}\left(e\left(\frac{j}{2 n}\right)+e\left(\frac{-j}{2 n}\right)\right)-1 \\
& =\frac{1-\cos (\pi / n)-\cos ((n+1) \pi / 2 n)+\cos ((n-1) \pi / 2 n)}{1-\cos (\pi / n)}-1 \\
& =\frac{\cos ((n-1) \pi / 2 n)-\cos ((n-1) \pi / 2 n)}{1-\cos (\pi / n)} \\
& =\csc \left(\frac{\pi}{2 n}\right) .
\end{aligned}
$$

\section{Proofs of Theorems}

$$
A=\operatorname{RFMLRcircfr}\left(e\left(\frac{0}{n}\right), e\left(\frac{1}{n}\right), e\left(\frac{3}{n}\right), \ldots, e\left(\frac{n-1}{n}\right)\right)
$$

The following is the proof of Theorem 1 .

is of the following form:

Proof. The matrix

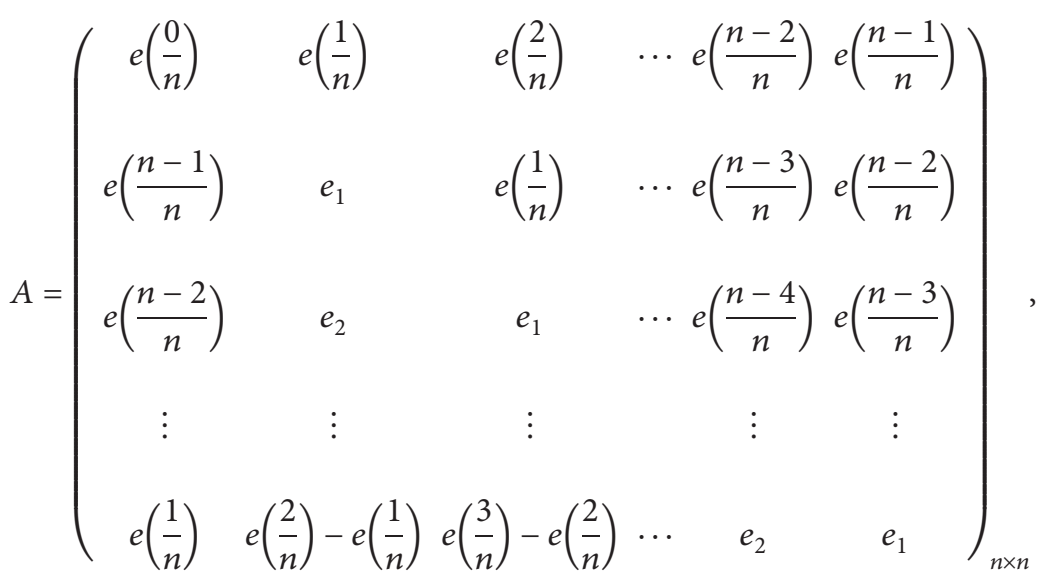

where

$$
\begin{aligned}
& e_{1}=e\left(\frac{0}{n}\right)-e\left(\frac{n-1}{n}\right), \\
& e_{2}=e\left(\frac{n-1}{n}\right)-e\left(\frac{n-2}{n}\right) .
\end{aligned}
$$

Using the definition of Frobenius norm and Lemma 1, $a_{k}=e(k / n)$, we have

$$
\begin{aligned}
\|A\|_{F}^{2}= & \sum_{k=0}^{n-1}\left|a_{k}\right|^{2}+(n-1)\left|a_{1}\right|^{2}+(n-2)\left|a_{2}\right|^{2}+\cdots+\left|a_{n-1}\right|^{2}+ \\
& (n-1)\left|a_{0}-a_{n-1}\right|^{2}+(n-2)\left|a_{n-1}-a_{n-2}\right|^{2}+\cdots+\left|a_{2}-a_{1}\right|^{2},
\end{aligned}
$$


and then $\left|a_{k}\right|=|e(k / n)|=1$,

$$
\begin{aligned}
\left|a_{0}-a_{n-1}\right|^{2} & =\left|e\left(\frac{0}{n}\right)-e\left(\frac{n-1}{n}\right)\right|^{2}=\left|e\left(\frac{0}{n}\right)-e\left(\frac{-1}{n}\right)\right|^{2}=\left|e\left(\frac{1}{n}\right)-1\right|^{2}, \\
\left|a_{n-1}-a_{n-2}\right|^{2} & =\left|e\left(\frac{n-1}{n}\right)-e\left(\frac{n-2}{n}\right)\right|^{2}=\left|e\left(\frac{-1}{n}\right)-e\left(\frac{-2}{n}\right)\right|^{2}=\left|e\left(\frac{1}{n}\right)-1\right|^{2}, \\
\left|e\left(\frac{1}{n}\right)-1\right|^{2} & =\left|\cos \left(\frac{2 \pi}{n}\right)-1+i \sin \left(\frac{2 \pi}{n}\right)\right|^{2}=4 \sin ^{2}\left(\frac{\pi}{n}\right),
\end{aligned}
$$

that is to say,

$$
\|A\|_{F}^{2}=\frac{n(n+1)}{2}+2 n(n-1) \sin ^{2}\left(\frac{\pi}{n}\right) .
$$

Using $\|A\|_{2} \geq(1 / \sqrt{n})\|A\|_{F}$, we can obtain the lower bound

$$
\|A\|_{2} \geq \sqrt{\frac{n+1}{2}+2(n-1) \sin ^{2}\left(\frac{\pi}{n}\right)} .
$$

In another case, let the matrices $Q_{1}, Q_{2}$, and $Q_{3}$ be defined by

$$
\begin{aligned}
Q_{1} & =\left(\begin{array}{cccccc}
0 & 1 & 0 & \cdots & 0 & 0 \\
0 & 0 & 1 & \cdots & 0 & 0 \\
0 & 0 & 0 & \cdots & 0 & 0 \\
\vdots & \vdots & \vdots & & \vdots & \vdots \\
0 & 0 & 0 & \cdots & 0 & 1 \\
1 & 0 & 0 & \cdots & 0 & 0
\end{array}\right)_{n \times n}, \\
Q_{2} & =\left(\begin{array}{cccccc}
0 & 0 & 0 & \cdots & 0 & 0 \\
0 & 0 & 0 & \cdots & 0 & 0 \\
0 & 1 & 0 & \cdots & 0 & 0 \\
\vdots & \vdots & \vdots & & \vdots & \vdots \\
0 & 0 & 0 & \cdots & 0 & 0 \\
0 & 0 & 0 & \cdots & 1 & 0
\end{array}\right)_{n \times n}, \\
Q_{3} & =\left(\begin{array}{cccccc}
0 & 0 & 0 & \cdots & 0 & 0 \\
0 & 1 & 0 & \cdots & 0 & 0 \\
0 & 0 & 1 & \cdots & 0 & 0 \\
\vdots & \vdots & \vdots & & \vdots & \vdots \\
0 & 0 & 0 & \cdots & 1 & 0 \\
1 & 0 & 0 & \cdots & 0 & 1
\end{array}\right)_{n \times n}
\end{aligned}
$$

$$
\begin{aligned}
\|A\|_{2} & =\left\|\sum_{k=0}^{n-1} a_{k} Q_{1}^{k}-\sum_{k=1}^{n-2} a_{n-k-1} Q_{2}^{k}-a_{n-1} Q_{3}\right\|_{2} \\
& \leq \sum_{k=0}^{n-1}\left|a_{k}\right|\left\|Q_{1}\right\|_{2}^{k}+\sum_{k=1}^{n-2}\left|a_{n-k-1}\right|\left\|Q_{2}\right\|_{2}^{k}+\left|a_{n-1}\right|\left\|Q_{3}\right\|_{2} .
\end{aligned}
$$

Since

$$
\begin{aligned}
Q_{1}^{H} Q_{1} & =\left(\begin{array}{cccc}
1 & 0 & \cdots & 0 \\
0 & 1 & \cdots & 0 \\
\vdots & \vdots & & \vdots \\
0 & 0 & \cdots & 1
\end{array}\right)_{n \times n}, \\
Q_{2}^{H} Q_{2} & =\left(\begin{array}{ccccc}
0 & 0 & \cdots & 0 & 0 \\
0 & 1 & \cdots & 0 & 0 \\
\vdots & \vdots & & \vdots & \vdots \\
0 & 0 & \cdots & 1 & 0 \\
0 & 0 & \cdots & 0 & 0
\end{array}\right)_{n \times n}, \\
Q_{3}^{H} Q_{3} & =\left(\begin{array}{cccc}
0 & 0 & \cdots & 0 \\
0 & 1 & \cdots & 0 \\
\vdots & \vdots & & \vdots \\
0 & 0 & \cdots & 1
\end{array}\right)_{n \times n} .
\end{aligned}
$$

Hence,

$$
\begin{aligned}
\left\|Q_{1}\right\|_{2} & =\left\|Q_{2}\right\|_{2}=\left\|Q_{3}\right\|_{2}=1, \\
\|A\|_{2} & \leq \sum_{k=0}^{n-1}\left|a_{k}\right|+\sum_{k=1}^{n-2}\left|a_{n-k-1}\right|+\left|a_{n-1}\right|,
\end{aligned}
$$

and by $\left|a_{k}\right|=|e(k / n)|=1,\|A\|_{2} \leq 2 n-1$; thus,

$$
\sqrt{\frac{n+1}{2}+2(n-1) \sin ^{2}\left(\frac{\pi}{n}\right)} \leq\|A\|_{2} \leq 2 n-1 .
$$

Then, we can obtain $A=\sum_{k=0}^{n-1} a_{k} Q_{1}^{k}-\sum_{k=1}^{n-2} a_{n-k-1} Q_{2}^{k}-a_{n-1} Q_{3}$, by identities of matrix norms,
This proves Theorem 1 .

Now, we prove Theorem 2. 
Proof

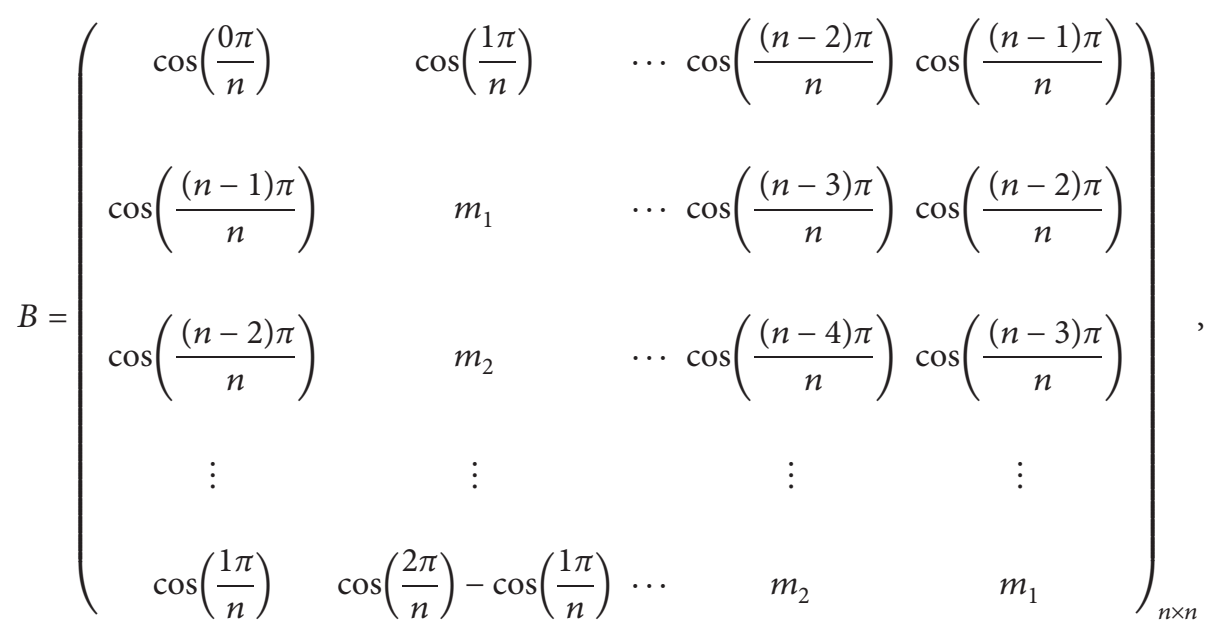

where

$$
\begin{aligned}
& m_{1}=\cos \left(\frac{0 \pi}{n}\right)-\cos \left(\frac{(n-1) \pi}{n}\right), \\
& m_{2}=\cos \left(\frac{(n-1) \pi}{n}\right)-\cos \left(\frac{(n-2) \pi}{n}\right) .
\end{aligned}
$$

Using the Frobenius norms and Theorem 1, as well as $b_{j}=\cos (j \pi / n)$, we have

$$
\begin{aligned}
\|B\|_{F}^{2} & =n \sum_{j=0}^{n-1} b_{j}^{2}+\sum_{j=1}^{n-1} j b_{j}^{2}-2 \sum_{j=1}^{n-2} j b_{j} b_{j+1}-2(n-1) b_{0} b_{n-1} \\
& =n \sum_{j=0}^{n-1} b_{j}^{2}+\sum_{k=1}^{n-1} \sum_{j=n-k}^{n-1} b_{j}^{2}-2 \sum_{k=1}^{n-2} \sum_{j=n-k-1}^{n-2} b_{j} b_{j+1}-2(n-1) b_{0} b_{n-1} \\
& =n \sum_{j=0}^{n-1} b_{j}^{2}+\sum_{k=1}^{n-1}\left(\sum_{j=0}^{n-1} b_{j}^{2}-\sum_{j=0}^{n-k-1} b_{j}^{2}\right)-2 \sum_{k=1}^{n-2}\left(\sum_{j=0}^{n-2} b_{j} b_{j+1}-\sum_{j=0}^{n-k-2} b_{j} b_{j+1}\right)-2(n-1) b_{0} b_{n-1} .
\end{aligned}
$$

By Lemma $1, \sum_{j=0}^{n-1} \cos ^{2}(j \pi / n)=(n / 2)$. Using the identities $\cos \theta=\left(e^{i \theta}+e^{-i \theta}\right) / 2$,

$$
e(x)=e^{2 \pi i x}
$$$$
\cos \left(\frac{(2 j+1) \pi}{n}\right)=\frac{e((2 j+1) / 2 n)+e(-(2 j+1) / 2 n)}{2}
$$ 
we can obtain

$$
\begin{aligned}
\sum_{j=0}^{n-1} \cos \left(\frac{(2 j+1) \pi}{n}\right) & =\frac{1}{2} \sum_{j=0}^{n-1}\left(e\left(\frac{2 j+1}{2 n}\right)+e\left(-\frac{2 j+1}{2 n}\right)\right) \\
& =\frac{1}{2}\left(\frac{e(1 / 2 n)(1-e(1))}{1-e(1 / n)}+\frac{e(1 / 2 n)(1-e(-1))}{1-e(-1 / n)}\right) \\
& =0
\end{aligned}
$$$$
\sum_{j=0}^{n-1} \cos \left(\frac{j \pi}{n}\right) \cos \left(\frac{(j+1) \pi}{n}\right)=\frac{1}{2} \sum_{j=0}^{n-1} \cos \left(\frac{2(j+1) \pi}{n}\right)+\frac{n}{2} \cos \left(\frac{\pi}{n}\right)
$$$$
=\frac{n}{2} \cos \left(\frac{\pi}{n}\right) \text {. }
$$

Hence,

$$
\sum_{j=0}^{n-2} \cos \left(\frac{j \pi}{n}\right) \cos \left(\frac{(j+1) \pi}{n}\right)=\frac{n-2}{2} \cos \left(\frac{\pi}{n}\right),
$$

and then, we can obtain

$\sum_{k=1}^{n-2} \sum_{j=0}^{n-k-2} \cos \left(\frac{j \pi}{n}\right) \cos \left(\frac{(j+1) \pi}{n}\right)=\frac{1}{8} \sec \left(\frac{\pi}{n}\right)+\frac{(n-1)(n-2)}{4}$.

\section{Therefore,}

$$
\begin{aligned}
B & =\sum_{j=0}^{n-1} b_{j} Q_{1}^{j}-\sum_{j=1}^{n-2} b_{n-j-1} Q_{2}^{j}-b_{n-1} Q_{3}, \\
\|B\|_{2} & =\left\|\sum_{j=0}^{n-1} b_{j} Q_{1}^{j}-\sum_{j=1}^{n-2} b_{n-j-1} Q_{2}^{j}-b_{n-1} Q_{3}\right\|_{2} \\
& \leq \sum_{j=0}^{n-1}\left|b_{j}\right|\left\|Q_{1}\right\|_{2}^{j}+\sum_{j=1}^{n-2}\left|b_{n-j-1}\right|\left\|Q_{2}\right\|_{2}^{j}+\left|b_{n-1}\right| \mid Q_{3} \|_{2} \\
& =\sum_{j=0}^{n-1}\left|b_{j}\right|+\sum_{j=1}^{n-2}\left|b_{n-j-1}\right|+\left|b_{n-1}\right| \\
& =\sum_{j=0}^{n-1}\left|\cos \left(\frac{j \pi}{n}\right)\right|+\sum_{j=1}^{n-2}\left|\cos \left(\frac{(n-j-1) \pi}{n}\right)\right|+\left|\cos \left(\frac{(n-1) \pi}{n}\right)\right| \\
& =\sum_{j=0}^{n-1}\left|\cos \left(\frac{j \pi}{n}\right)\right|+\sum_{j=1}^{n-2}\left|\cos \left(\frac{(j+1) \pi}{n}\right)\right|+\left|\cos \left(\frac{\pi}{n}\right)\right| \\
& =2 \sum_{j=0}^{n-1}\left|\cos \left(\frac{j \pi}{n}\right)\right|-1 .
\end{aligned}
$$


By Lemma 3, if $n$ is even,

$$
\sum_{j=0}^{n-1}\left|\cos \left(\frac{j \pi}{n}\right)\right|=\cot \left(\frac{\pi}{2 n}\right) .
$$

Therefore, if $n$ is even, $\|B\|_{2} \leq 2 \cot (\pi / 2 n)-1$. If $n$ is odd,

$$
\sum_{j=0}^{n-1}\left|\cos \left(\frac{j \pi}{n}\right)\right|=\csc \left(\frac{\pi}{2 n}\right) .
$$

Therefore, if $n$ is odd, $\|B\|_{2} \leq 2 \csc (\pi / 2 n)-1$.

This proves Theorem 2 .

Now, we prove Theorem 3.

$$
C=\left\{\begin{array}{c}
\sin \left(\frac{0 \pi}{n}\right) \\
\sin \left(\frac{(n-1) \pi}{n}\right) \\
\sin \left(\frac{(n-2) \pi}{n}\right) \\
\vdots \\
\sin \left(\frac{1 \pi}{n}\right) \\
\sin \left(\frac{2 \pi}{n}\right.
\end{array}\right),
$$

Using the Frobenius norms and Theorem 1, as well as $c_{j}=\sin (j \pi / n)$, we have

$$
\begin{aligned}
\|C\|_{F}^{2}= & n \sum_{j=0}^{n-1} c_{j}^{2}+\sum_{j=1}^{n-1} j c_{j}^{2}-2 \sum_{j=1}^{n-2} j c_{j} c_{j+1}-2(n-1) c_{0} c_{n-1} \\
= & n \sum_{j=0}^{n-1} c_{j}^{2}+\sum_{k=1}^{n-1} \sum_{j=n-k}^{n-1} c_{j}^{2}-2 \sum_{k=1}^{n-2} \sum_{j=n-k-1}^{n-2} c_{j} c_{j+1} \\
= & n \sum_{j=0}^{n-1} c_{j}^{2}+\sum_{k=1}^{n-1}\left(\sum_{j=0}^{n-1} c_{j}^{2}-\sum_{j=0}^{n-k-1} c_{j}^{2}\right) \\
& -2 \sum_{k=1}^{n-2}\left(\sum_{j=0}^{n-2} c_{j} c_{j+1}-\sum_{j=0}^{n-k-2} c_{j} c_{j+1}\right) .
\end{aligned}
$$

By Lemma $1, \sum_{j=0}^{n-1} \sin ^{2}(j \pi / n)=(n / 2)$. Using the identities

$$
\begin{aligned}
\cos \theta & =\frac{e^{i \theta}+e^{-i \theta}}{2}, \\
e(x) & =e^{2 \pi i x}, \\
\sum_{j=0}^{n-2} \sin \left(\frac{j \pi}{n}\right) \sin \left(\frac{(j+1) \pi}{n}\right) & =\frac{1}{2} \sum_{j=0}^{n-2}\left(\cos \left(\frac{\pi}{n}\right)-\cos \left(\frac{2(j+1) \pi}{n}\right)\right) \\
& =\frac{n}{2} \cos \left(\frac{\pi}{n}\right),
\end{aligned}
$$

and by Theorem 1, we can obtain

$$
\begin{aligned}
& \sum_{k=1}^{n-2} \sum_{j=0}^{n-k-2} \sin \left(\frac{j \pi}{n}\right) \sin \left(\frac{(j+1) \pi}{n}\right) \\
& =\sum_{k=1}^{n-2} \frac{n-k-1}{2} \cos \left(\frac{\pi}{n}\right)-\frac{1}{2} \sum_{k=1}^{n-2} \sum_{j=0}^{n-k-2} \cos \left(\frac{2(j+1) \pi}{n}\right) \\
& =\frac{n(n-2)}{2} \cos \left(\frac{\pi}{n}\right)-\frac{1}{16} \sec \left(\frac{\pi}{n}\right)-\frac{(n-1)(n-2)}{8} .
\end{aligned}
$$


Therefore,

$$
\|C\|_{F}^{2}=\frac{2 n^{2}+3 n-2}{4}-\frac{1}{8} \sec \left(\frac{\pi}{n}\right) .
$$

We can obtain $\|C\|_{2} \geq(1 / \sqrt{n})\|C\|_{F}=\sqrt{(\beta / n)}$, where

$$
\beta=\frac{2 n^{2}+3 n-2}{4}-\frac{1}{8} \sec \left(\frac{\pi}{n}\right) \text {. }
$$

In another case, using Theorem 1 , and for the matrices $Q_{1}, Q_{2}$, and $Q_{3}$ as mentioned above, by $c_{j}=\sin (j \pi / n) \geq 0$, $\left\|Q_{1}\right\|_{2}=\left\|Q_{2}\right\|_{2}=\left\|Q_{3}\right\|_{2}=1$. We have

$$
\begin{aligned}
C & =\sum_{j=0}^{n-1} c_{j} Q_{1}^{j}-\sum_{j=1}^{n-2} c_{n-j-1} Q_{2}^{j}-c_{n-1} Q_{3}, \\
\|C\|_{2} & =\left\|\sum_{j=0}^{n-1} c_{j} Q_{1}^{j}-\sum_{j=1}^{n-2} c_{n-j-1} Q_{2}^{j}-c_{n-1} Q_{3}\right\|_{2} \\
& \leq \sum_{j=0}^{n-1}\left|c_{j}\right|\left\|Q_{1}\right\|_{2}^{j}+\sum_{j=1}^{n-2}\left|c_{n-j-1}\right|\left\|Q_{2}\right\|_{2}^{j}+\left|c_{n-1}\right|\left\|Q_{3}\right\|_{2} \\
& =\sum_{j=0}^{n-1}\left|c_{j}\right|+\sum_{j=1}^{n-2}\left|c_{n-j-1}\right|+\left|c_{n-1}\right| \\
& =\sum_{j=0}^{n-1} \sin \left(\frac{j \pi}{n}\right)+\sum_{j=1}^{n-2} \sin \left(\frac{(n-j-1) \pi}{n}\right)+\sin \left(\frac{(n-1) \pi}{n}\right) \\
& =2 \sum_{j=0}^{n-1} \sin \left(\frac{j \pi}{n}\right) \\
& =2 \cot \left(\frac{\pi}{2 n}\right) .
\end{aligned}
$$

Thus, the result is obtained as follows:

$$
\sqrt{\frac{\beta}{n}} \leq\|C\|_{2} \leq 2 \cot \left(\frac{\pi}{2 n}\right),
$$

where

$$
\beta=\frac{2 n^{2}+3 n-2}{4}-\frac{1}{8} \sec \left(\frac{\pi}{n}\right) .
$$

This completes all of the theorems.

\section{Conclusion}

The spectral norms of RFMLR-circulant matrices involving exponential forms and trigonometric functions are investigated in this paper. The computation complexity of this paper is lower than the previous work. By using the algorithms of this paper, we can further study the identities of $f(x)$-circulant matrices, such as RFPrLrR-cieculant matrix and RFMLrR-circulant matrix. Simulation is very important for applications of our work, and these will be our further topics to study.

\section{Data Availability}

The data used to support the findings of this study are available from the corresponding author upon request.

\section{Conflicts of Interest}

The author declares conflicts of interest.

\section{Authors' Contributions}

The author contributed to each part of this work seriously and read and approved the final version of the manuscript.

\section{Acknowledgments}

This work was supported by NSF (11771351).

\section{References}

[1] Y. Wang, B. Niu, H. Wang, N. Alotaibi, and E. Abozinadah, "Neural network-based adaptive tracking control for switched nonlinear systems with prescribed performance: an average dwell time switching approach," Neurocomputing, vol. 435, pp. 295-306, 2021.

[2] S. Solak, "On the norms of circulant matrices with the Fibonacci and Lucas numbers," Applied Mathematics and Computation, vol. 160, no. 1, pp. 125-132, 2005.

[3] S. Shen and J. Cen, "On the bounds for the norms of $r$-circulant matrices with the Fibonacci and Lucas numbers," Applied Mathematics and Computation, vol. 216, no. 10, pp. 2891-2897, 2010.

[4] Y. Yazlik and N. Taskara, "On the norms of an $r$-circulant matrix with the generalized $k$-Horadam numbers," Journal of Inequalities and Applications, vol. 2013, no. 1, Article ID 394, 2013.

[5] C. Kizilates and N. Tuglu, "On the bounds for the spectral norms of geometric circulant matrices," Journal of Inequalities and Applications, vol. 312, no. 3, pp. 2-9, 2016.

[6] N. Tuglu and C. Kizilates, "On the norms of circulant and $r$ circulant matrices with the hyperharmonic Fibonacci numbers," Journal of Inequalities and Applications, vol. 253, pp. 11-18, 2015.

[7] H. Gökbas and R. Turkmen, "On the norms of $r$-Toeplitz matrices involving Fibonacci and Lucas numbers," Advances in Linear Algebra and Matrix Theory, vol. 6, pp. 31-39, 2016.

[8] H. Gökbas and H. Köse, "On the norms of $r$-hankel matrices involving Fibonacci and Lucas numbers," Journal of Applied Mathematics and Physics, vol. 7, no. 6, pp. 1409-1417, 2018.

[9] B. J. Shi, "On the spectral norms of some circulant matrices with the trigonometric functions," Journal of Inequalities and Applications, vol. 225, pp. 1-9, 2019.

[10] B. J. Shi, "On the norms of $r$-Hankel and $r$-Toeplitz matrices," Mathematical Problems in Engineering, vol. 2019, Article ID 6729701, 4 pages, 2019.

[11] Z. L. Jiang, N. Shen, and J. Li, "Determinants of the RFMLR circulant matrices with perrin, padovan, tribonacci, and the generalized Lucas numbers," Journal of Applied Mathematics, vol. 2014, Article ID 585438, 11 pages, 2014. 